\title{
Training in healthcare during and after COVID-19: proposal for simulation training
}

\author{
Carolina Felipe Soares Brandão 1* (D), Ellen Cristina Bergamasco² (D), Gabriela Furst Vaccarezza ${ }^{3}$ (1), \\ Maria Luiza Ferreira de Barba ${ }^{4}$ (1) Enrico Ferreira Martins de Andrade ${ }^{5}$ (D), Dario Cecilio-Fernandes ${ }^{6}$ (1)
}

\section{SUMMARY}

INTRODUCTION: The challenge of facing COVID-19 falls under all health care structures, and without specific training to health care professionals they are probably the professionals with the highest level of exposure. Regardless of the level of health care, the training of professionals aims to optimize resources and attend patients while assuring quality and security.

POINT OF VIEW: This report proposes simulation training for health care professionals to update professionals for attending patients during the pandemic. This training was built with five simulated stations, considering different stages of a patient with COVID-19. This report takes advantage of different simulation techniques, such as skills training, standardized patient, medium- and high-fidelity simulator, rapid cycle of deliberate practice, and in situ simulation.

DISCUSSION: Medical procedures for COVID-19 patients offer additional risk for health care professionals, especially considering exposure to procedures that generate aerosols, such as compression, mask ventilation, and orotracheal intubation. Thus, finding educational strategies that allow training is essential to simulate the evolution of COVID-19 patients in a safe manner.

CONCLUSION: Simulation has proven to be a useful and effective form of training around the world for training health teams on the front lines for patient care in COVID-19.

KEYWORDS: Coronavirus infections. Simulation technique. High-fidelity simulation training. Simulation training.

The challenge of the COVID-19 pandemic is felt in all health care spheres and will certainly persist after the pandemic. There is no doubt that, without specific training, medical professionals will be among those most exposed to illnesses and, consequently, to sick leave, with loss to the team and society. Regardless of the health care level at which professionals work, training aims to optimize resources and promote quality care while ensuring the safety of the professionals and the population.
In this study, we propose a continuing education model for professional development related to the treatment of patients with suspected or confirmed COVID-19 cases. This model is based on simulation training that aims to educate health professionals on COVID-19 protocols.

In general, simulations can be contextualized as educational strategies that range form practicing basic skills to the actual professional treatment of a clinical case. In the latter, technical

\footnotetext{
'Universidade Municipal de São Caetano, Faculty of Medicine, Laboratório de Simulação - São Caetano do Sul (SP), Brazil.

${ }^{2}$ Faculdade Israelita de Ciências da Saúde Albert Einstein - São Paulo (SP), Brazil.

${ }^{3}$ Universidade Municipal de São Caetano do Sul, Faculty of Medicine - São Caetano do Sul (SP), Brazil.

${ }^{4}$ Universidade Estácio de Sá - Rio de Janeiro (RJ), Brazil.

5Universidade Municipal de São Caetano do Sul, Faculty of Medicine - São Caetano do Sul (SP), Brazil.

${ }^{6}$ Universidade Estadual de Campinas - Campinas (SP), Brazil.

*Corresponding author: carolinafs11@gmail.com

Conflicts of interest: The authors declare there are no conflicts of interest. Funding: The author Dario Cecilio-Fernandes is partially funded by FAPESP São Paulo Research Foundation [Young Investigator Grant number 2018/15642-1 and Regular Grant number 2020/04909-7].

Received on August 20, 2020. Accepted on September 20, 2020.
} 
and nontechnical skills (soft skills) are practiced simultaneously. One of the most widespread definitions of simulation states it should be seen as a technique, and not exclusively a technology, which aims to replace or amplify a real experience with supervision, but substantially evokes aspects of the real world in an interactive environment ${ }^{1}$. The use of simulations is no longer considered an innovative activity, given there are reports and records dating back to more than 100 years of technical skills training, using straw-filled dummies ${ }^{2}$. In recent decades, simulations have been updated from both a pedagogical and a technological point of view, such as with robots that confer high fidelity in real time to the actions performed with the relevant hemodynamics, enabling the creation of scenarios more similar to real-world practice.

This technological advance has allowed the training of competencies, such as decision-making, leadership, teamwork, and other determinant actions for better patient care. This strategy can simultaneously work from several aspects desirable to health professionals, and can be included in curricula and in continuing education, according to the available time and financial resources and the target audience to be trained. Training that addresses the management of patients with COVID-19 has been conducted worldwide. Among the main strategies used, simulation stands out, because it ensures a safe environment and prepares for care under situations with the potential risk of contagion ${ }^{3}$.

The novel coronavirus (SARS-CoV-2), which causes COVID19 , belongs to the family of betacoronaviruses, RNA viruses that adapt to various hosts and spread rapidly among humans. There are many uncertainties about the management of the disease, but it is known that the contamination of professionals through aerosols and fomites is highly likely, and a high incidence of fecal transmission has been described among professionals responsible for direct patient care ${ }^{4}$. The clinical presentation of this disease is mostly mild, with approximately $80 \%$ of infected patients not needing any special care, and the symptoms are similar to those of the common flu; however, a portion of patients can progress to a more severe disease from, with respiratory failure as one of the most important clinical signs ${ }^{5}$.

The big question is how to train these professionals to effectively cope with the ongoing pandemic and how to maintain this standard after this situation is under control. Additionally, considering that many health professionals are inexperienced with this disease is important, making it necessary to focus their training on specific changes for the care of patients with suspected COVID-19. In this context, many videos using simulated patients have been employed as a practical strategy to disseminate at least the minimum content and requirements for care. However, these isolated activities may garner too little participant engagement and do not promote the hands-on experience needed by professionals who perform numerous procedures, such as medical professionals, especially on the front line and in intensive care units (ICUs).

The COVID-19 pandemic caused many institutions to take a new perspective on simulation training, which ceased to be just another strategy and became the strategy of choice, as institutions made the necessary adjustments to adapt to the pandemic ${ }^{6}$. Simulation programs were adjusted to meet health needs in order to ensure that teams would adapt to the crisis, since this teaching strategy enables improvements in individual and team learning.

Procedures including endotracheal intubation, collection of laboratory samples ${ }^{7}$, collection of tracheal secretions ${ }^{8}$, and cardiopulmonary resuscitation $(\mathrm{CPR})^{9,10}$ have been used in training to develop skills and ensure the safety of teams ${ }^{7}$ with respect to COVID-19. Training in donning and doffing in the context of COVID-19 using rapid cycle deliberate practice has also been planned in the pandemic ${ }^{11}$. Training using simulation improves the perceived confidence and preparation of professionals with regard to patients diagnosed with COVID-197

To design an adequate simulation training program, understanding three fundamental concepts is required: fidelity, realism, and complexity. The concept of fidelity refers to the amount of technology used in robots or manikins and can be classified as low, medium, and high ${ }^{12-14}$. With low-fidelity simulators, it is possible to train a motor skill; medium-fidelity simulators combine part of the manikin with software to train on a specific procedure; and high-fidelity simulators are those with spontaneous breathing and real-time hemodynamic changes, allowing training for advanced clinical situations using a simulator, in which different procedural interventions are possible simultaneously. Whereas fidelity refers to the manikin or simulator, realism is related to how close the scenario or clinical case is to reality. Realism is traditionally divided into four domains: conceptual, physical, functional, and psychological ${ }^{15-19}$. The concept of complexity is related to the degree of difficulty of the scenario, recalling that the same scenario can be complex for a group of professionals (e.g., undergraduate students) and less complex for another group (experienced professionals). To develop a simulation training program, considering all available resources is of utmost importance, as well as the learning objectives in order to optimize the simulation usefulness ${ }^{20}$.

The aim of this report is to propose a simulation-based training program focused on patient and professional safety during and after the COVID-19 pandemic. This proposal is based on the combination of the fundamental concepts of simulation with the learning objectives of each training exercise. 


\section{SIMULATION TRAINING FOR COVID-19}

The goal of the training program involves different types of simulation that may or may not occur simultaneously in the rotation scheme, depending on the number of professionals to be trained and on instructors trained in the method and subject, as well as on room availability. Eight to 12 professionals are expected to participate in each station. The duration includes the scenario time and the time that will be used for feedback/ debriefing; for the proposed program, approximately $30 \mathrm{~min}$ utes is suggested for each case. If the program is performed with only one group of participants and sequential stations, the time at each station may vary depending on its difficulty to the group. When the program is performed with more groups and stations occurring simultaneously in a rotation model, it is important to keep the activity time equal for all.

The proposal considers five stations, starting with basic procedures and gradually evolving with the patient's complexity (see Table 1). The stations start with the donning, doffing, and use of personal protective equipment (PPE). After that, groups proceed to the screening of the patient with suspected COVID19 , followed by critically ill patient care and CPR, and ending with the pronation of the critically ill patient.

The first station aims to practice fundamental technical skills during and after the COVID-19 pandemic: donning, doffing, and use of PPE. For this activity, the use of the skills training model can fulfill the proposal. At this point, the objective is the procedural process, and there is no need for a particular description of a clinical case. The facilitator of this activity should accurately know the resources available in the routine of these professionals and master the care practices of the institution. All participants must perform the complete technical procedure individually so that they can receive immediate feedback and repeat the activity until correct - essential to ensure the effectiveness of the activity. A simple room and a table containing the material to be used (alcohol gel, goggles, mask, procedure glove, apron, hazardous waste bag or container for disposal of the material used) are the necessary materials. A clipboard, paper, and pen can be made available to participants to take notes at this and other stations.

For the second station, we considered the moment of triage of a patient with suspected COVID-19, a situation commonly faced in health services. The use of a standardized patient ensures the fidelity of the scenario and allows the participant to perform a rapid anamnesis, ask the right focused questions, identify signs and symptoms, and engage in clinical reasoning and decision-making. The construction of a clinical case with all the patient's previous and current data and information is mandatory for this activity. The performance of a pilot exercise with an actor who will represent the standardized patient is essential to emphasize the objective of the activity and the attitudes to be taken during the scenario to ensure that the representation is homogeneous between all the groups being trained. This type of simulation is widely used when the objective is to learn technical content and practice interaction and communication between provider and patient. In this model, it is suggested that only one participant act as a volunteer for care, while the others act as observers. Considering good triage practices, a 5-minute scenario may be considered, and at the end, everyone should participate in the debriefing. In times of the pandemic and even after the acute phase of COVID-19, developing scenarios that foster discussion of clinical reasoning and decision-making is essential, in addition to the use of PPE and aspects of patient/professional safety. The clinical history may

Table 1. Simulation training stations for technical and non-technical skills, focused on patient safety during and after the COVID-19 pandemic.

\begin{tabular}{l|c|c|c}
\hline Station 1 & Lonning, doffing, and use of PPE* & Technical skills training & Materials for specific training (PPE) \\
\hline Station 2 & $\begin{array}{c}\text { Screening of suspected patient } \\
\text { with COVID-19 }\end{array}$ & $\begin{array}{c}\text { Clinical reasoning and technical } \\
\text { skills training }\end{array}$ & Standardized patient \\
\hline Station 3 & $\begin{array}{c}\text { Triage of critically ill patient with } \\
\text { COVID-19 }\end{array}$ & $\begin{array}{c}\text { Clinical reasoning and technical } \\
\text { and non-technical skills training }\end{array}$ & $\begin{array}{c}\text { Medium- or high-fidelity } \\
\text { simulator combined with } \\
\text { standardized patient }\end{array}$ \\
\hline Station 4 & CPR of the COVID-19patient & $\begin{array}{r}\text { Clinical reasoning and technical } \\
\text { skills training }\end{array}$ & Medium- or high-fidelity simulator \\
\hline Station 5 & $\begin{array}{c}\text { Care of critically ill patient with } \\
\text { COVID-19- Patient pronation in } \\
\text { critical care }\end{array}$ & $\begin{array}{c}\text { Clinical reasoning and technical } \\
\text { skills training }\end{array}$ & $\begin{array}{c}\text { Medium- or high-fidelity simulator } \\
\text { in the ICU of the institution }\end{array}$ \\
\hline
\end{tabular}

*PPE: personal protective equipment 
change according to the level of knowledge of the team to be trained, as well as the expected behaviors. A room containing a table, chairs, material for checking vital signs, PPE, alcohol gel, and garbage for disposal of contaminated material are the necessary resources.

The third station aims to simulate critical patient care. In this context, a medium- or high-fidelity simulator is suggested, in which the interventions performed by the health team should be discussed, such as proper donning of equipment; identification of the patient with signs and symptoms of clinical decompensation; checking and administering medications, the permeability of venous access, and medical records; clinical interpretation of laboratory and imaging findings, checking hemodynamic signs; and requesting intervention by other professionals. The construction of a clinical case and the performance of the pilot exercise are necessary to verify whether the information and clues offered in the case align with what is expected during the real scenario. This type of scenario allows participants to be trained in the knowledge of signs of clinical worsening, the application of care protocols, and the practicing of teamwork. It is suggested that two or more participants be volunteers in the scenario, according to the objective defined in the case. The scenario may last 10 minutes or more. At the end of care, volunteers and observers will participate in the debriefing. Creating a checklist to direct the observation of participants and conduct the debriefing is recommended. In this scenario, there is the possibility of including an actor to simulate a companion, providing the participants with an opportunity to discuss technical and attitudinal knowledge. To train the actor who will represent the companion, it is important to make it clear that this actor should avoid caricatured or exacerbated actions that do not contribute to teaching and that push the professionals away from the objective of the scenario. For this type of scenario, it is necessary to establish an inpatient unit containing a stretcher, material for care, and a complete emergency cart.

The fourth station aims to improve the identification and management of cardiac arrest and the performance of CPR maneuvers. We suggest using the rapid cycle deliberate practice educational strategy, which uses the concepts of simulation-based mastery learning, a strategy widely validated in the literature that is based on the need for demonstration of the student's proficiency in a competency before moving to the next educational objective, aiming at mastering a given competency ${ }^{6}$. A medium- or high-fidelity simulator is suggested herein so that resuscitation interventions can be performed. The group of participants can be divided into three or four small groups that will perform the identification of cardiac arrest and CPR maneuvers. All groups should perform the activities, rotating between practice and observation. In this station, there will be no debriefing after care; however, it is up to the facilitator to interrupt care, provide feedback, and direct the proper performance of the techniques. This is an intentional strategy that works well for this type of scenario in which there are many demands (compression, ventilation, rhythm identification, others). All participants must practice compression and ventilation during the time scheduled for this station. Few resources are used; they should include a room for care, simulator, rigid board, bag-mask valve, defibrillator, PPE, alcohol gel, and garbage for disposal of material.

The fifth station aims to train the participants in the care provided for pronation of the critically ill patient with COVID19. Critically ill patients with COVID-19 may require placement in the prone position to improve ventilation ${ }^{8}$. Professionals should be carefully trained in this procedure because it can cause numerous serious adverse events, such as extubating and loss of central venous access. Our proposal suggests the in situ simulation, which is more complex, given the logistics of care and the many material resources needed. Figure 1 shows an example of a ward to be set up for this type of simulation. This type of simulation occurs in the actual care environment and can train the entire team as long as they are staggered in their work shifts. It is undoubtedly a motivating format because the training takes place using real resources and with the routine staff. Debriefing in this proposed method is essential for better knowledge retention. For this activity, it is essential to construct a clinical scenario and conduct the pilot exercise to assess whether the case and the clues provided direct the participants to the expected goal. The simulation scenario is suggested to last for 10 minutes on average, and the debriefing should happen afterward, using a checklist. Regarding the number of

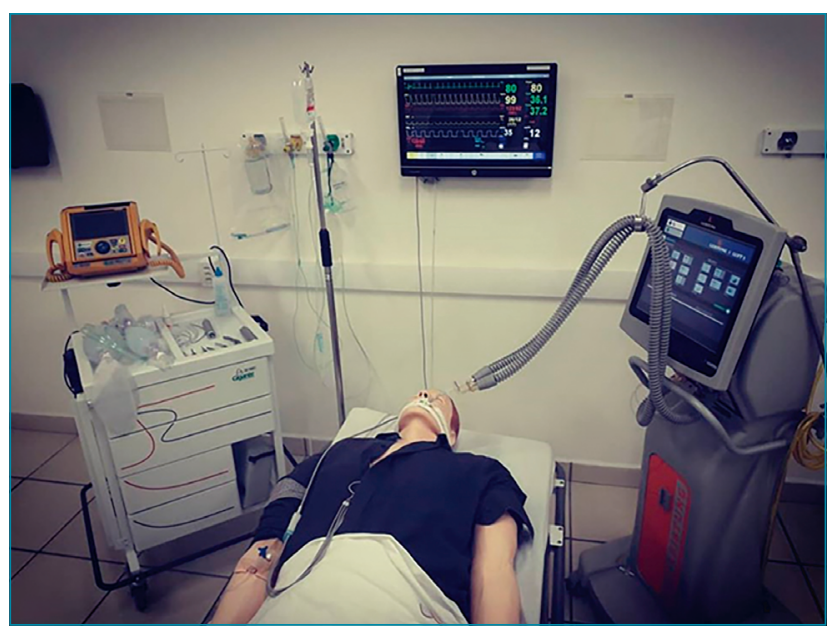

Figure 1. Example of a simulation of critical care for COVID-19 patients. 
participants, each institution should consider the number of professionals who will participate in the routine. The in situ simulation can be performed with a medium or high fidelity simulator or a standardized patient.

The five training stations proposed can be adapted according to the infrastructure of each service. We suggest constructing the cases and discussing them with colleagues to identify the fundamental objectives to be addressed in each activity. Checklists can help participants and facilitators during the activities, direct the debriefing, and serve as a reference material to be used at later times. Training the facilitator to conduct the debriefing is fundamental for knowledge retention. Audio-visual resources to record the sessions and incorporate them into the discussion is desirable, but their lack should not be an obstacle to the performance of the training exercises.

\section{DISCUSSION}

COVID-19 is a highly transmissible disease that directly affects health professionals, especially doctors and nurses working in direct patient care. A $20 \%$ contamination rate has been found in health professionals in several countries, and it is higher when they have not been prepared or did not use PPE properly $^{21}$. Different educational strategies have been put to the test during the COVID-19 pandemic, since it is necessary to consider educational measures that minimize biopsychosocial impacts but entail hands-on training performed in a controlled environment so that they, in fact, have a transformative and multiplier effect on patient and provider safety.

The growing demand of the population for access to health services requires new ways of organizing work processes so that the social demand for quality services can be met and the limitations imposed by the COVID-19 pandemic be overcome. Therefore, health becomes an essential arena for human development, with a greater awareness of its importance in the social and economic context.

The use of different simulation strategies can effectively contribute to the training of health professionals, especially physicians, at all health care levels. Educational institutions should offer, as much as possible, specific training to their care partners. Developing training programs in situations such as the COVID-19 pandemic promotes safety not only for patients but also for the professionals involved. Institutions that do not have simulation environments can adapt other ways of disseminating information in a didactic way, which will help health professionals in this difficult time that has impacted the entire world.

The simulation training program proposed in this study considered the progression of patients with COVID-19 and was concerned with training professionals in safe and adequate care. Training of basic activities such as donning, doffing, and using PPE, although simple, is essential for the safety of professionals and the prevention of cross-contamination. Training on $\mathrm{CPR}$ maneuvers was included in our program because these maneuvers for patients with COVID-19 pose additional risks to health professionals, especially if we consider the exposure to aerosols generated by procedures such as compression, mask ventilation, and orotracheal intubation. Training procedures to avoid the contamination of professionals are of vital importance at this time, and simulations consistently contribute to training in this pandemic times. Some studies have even used fluorescent powder to visually represent the virus in training on orotracheal intubation ${ }^{22}$.

The perception of professionals regarding safety in the care of patients with COVID-19 may increase after conducting simulation training; professionals may feel more confident and willing to care for patients with COVID-19 or other infectious diseases that require isolation ${ }^{7}$. A study that used in situ simulation to train ICU/critical-care doctors and nurses on the scenario of a patient with COVID-19 who started with respiratory distress, progressed to pulseless electrical activity secondary to hypoxemia, and ended with a code blue, concluding that there was improvement in the speed of the main events of the CPR maneuvers after the simulation sessions ${ }^{10}$. The authors also reported that observations of the in situ simulation allowed them to make changes to procedure as well as adjustments to the flow of care and infection prevention measures ${ }^{10}$.

\section{FINAL CONSIDERATIONS}

Simulation is a strategy that promotes reflection in a controlled environment that closely resembles the real-world environment. It is not a strategy to be used alone, but as a practical tool, since it helps exponentially in the development of health professionals, such as doctors and nurses. The improvement of the knowledge and skills of these professionals requires strategies that are not fragmented but consider the clinical reasoning and connection with the whole.

In the COVID-19 pandemic, providing professionals with training to develop analytical and reflective critical thinking is mandatory, focusing on not only technical aspects but also attitudinal and social aspects at different health care levels. Simulation is useful and effective in all parts of the world for the training of front-line healthcare teams to care for patients with COVID-19.

Building and implementing a training program that includes the basic aspects of donning PPE to the care of critically ill patients can help in the care of COVID-19 patients, improve the performance of professionals, and lead to better clinical outcomes. 


\section{AUTHORS" CONTRIBUTION}

CFSB: Conceptualization, Methodology, Formal Analysis, Supervision, Writing - Original Draft, Writing - Review \& Editing. ECB: Conceptualization, Methodology, Writing - Original Draft, Writing - Review \& Editing. GFV: Conceptualization, Methodology, Writing - Original
Draft, Writing - Review \& Editing. MLFB: Conceptualization, Methodology, Writing - Original Draft, Writing - Review \& Editing. EFMA: Conceptualization, Methodology, Writing - Original Draft, Writing - Review \& Editing. DCF: Conceptualization, Funding Acquisition, Methodology, Writing - Original Draft, Writing - Review \& Editing.

\section{REFERENCES}

1. Gaba DM. Do as we say, not as you do: using simulation to investigate clinical behavior in action. Simul Healthc. 2009;4(2):67-9. https://doi.org/10.1097/ SIH.0b013e3181a4a412

2. Nehring WM, Lashley FR. Nursing simulation: a review of the past 40 years. Simul Gaming. 2009;40(4):528-52. https://doi. org/10.1177/1046878109332282

3. Mohammadi G, Tourdeh M, Ebrahimian A. Effect of simulationbased training method on the psychological health promotion in operating room students during the educational internship. J Educ Health Promot. 2019;8:172. https://doi.org/10.4103/ jehp.jehp_106_19

4. Amirian ES. Potential fecal transmission of SARS-CoV-2: current evidence and implications for public health. Int J Infect Dis. 2020;95:363-70. https://doi.org/10.1016/j.ijid.2020.04.057

5. Brazil. Ministério da Saúde, Secretaria de Atenção Primária à Saúde (SAPS). Protocolo de manejo clínico do coronavírus (COVID-19) na atenção primária à saúde. Brasília: Ministério da Saúde; 2020. [cited on Aug. 03, 2020]. Available from: https://portalarquivos.saude.gov.br/images/pdf/2020/ April/07/20200407-ProtocoloManejo-ver07.pdf

6. Brydges R, Campbell DM, Beavers L, Khodadoust N, lantomasi $P$, Sampson K, et al. A lessons learned in preparing for and responding to the early stages of the COVID-19 pandemic: one simulation's program experience adapting to the new normal. Adv Simul (Lond). 2020;5:8. https://doi.org/10.1186/ s41077-020-00128-y

7. Khan JA, Kiani MRB. Impact of multi-professional simulationbased training on perceptions of safety and preparedness among health workers caring for coronavirus disease 2019 patients in Pakistan. J Educ Eval Health Prof. 2020;17:19. https://doi.org/10.3352/jeehp.2020.17.19

8. Mark ME, LoSavio P, Husain I, Papagiannpoulos P, Batra PS, Tajudeen BA. Effect of implementing simulation education on health care worker comfort with nasopharyngeal swabbing for COVID-19. Otolaryngol Head Neck Surg. 2020;163(2):271-4. https://doi.org/10.1177/0194599820933168

9. Lim WY, Wong P, Teo LM, Ho VK. Resuscitation during the COVID-19 pandemic: lessons learnt from high-fidelity simulation. Resuscitation. 2020;152:89-90. https://doi. org/10.1016/j.resuscitation.2020.05.024

10. Foong TW, Hui Ng ES, Wee Khoo CY, Ashokka B, Khoo D, Agrawal R. Rapid training of healthcare staff for protected cardiopulmonary resuscitation in the COVID-19 pandemic. Br J Anaesth. 2020;125(2):e257-9. https://doi.org/10.1016/j. bja.2020.04.081
11. Oliveira HC, Souza LC, Leite TC, Campos JF. Personal protective equipment in the coronavirus pandemic: training with rapid cycle deliberate practice. Rev Bras Enferm. 2020;73(Suppl 2):e20200303. https://doi.org/10.1590/0034-7167-2020-0303

12. Maran NJ, Glavin RJ. Low - to high-fidelity simulation - a continuum of medical education? Med Educ. 2003;37(Suppl 1):22-8. https://doi.org/10.1046/j.1365-2923.37.s1.9.x

13. Hamstra SJ, Brydges R, Hatala R, Zendejas B, Cook DA. Reconsidering fidelity in simulation-based training. Acad Med. 2014;89(3):387-92. https://doi.org/10.1097/ ACM.0000000000000130

14. Brandão CFS, Collares CF, Cecilio-Fernandes D. Simuladores, pacientes padronizados e híbridos. In: Scalabrini Neto A, Fonseca A, Brandão CFS, eds. Simulação realística e habilidades na saúde. Rio de Janeiro: Editora Atheneu; 2017. p.11-22.

15. Dieckmann P, Gaba D, Rall M. Deepening the theoretical foundations of patient simulation as social practice. Simul Healthc. 2007;2(3):183-93. https://doi.org/10.1097/SIH.0b013e3180f637f5

16. Rudolph JW, Simon R, Raemer DB. Which reality matters? Questions on the path to high engagement in healthcare simulation. Simul Healthc. 2007;2(3):161-3. https://doi. org/10.1097/SIH.0b013e31813d1035

17. Chiniara G, Cole G, Brisbin K, Huffman D, Cragg B, Lamacchia $M$, et al. Guidelines Working Group. Simulation in healthcare: a taxonomy and a conceptual framework for instructional design and media selection. Med Teach. 2013;35(8):e138095. https://doi.org/10.3109/0142159X.2012.733451

18. Dieckmann P, Rall M. Designing a scenario as a simulated clinical experience: the TuPASS scenario script. In: Kyle Jr. RR, Murray $W B$, eds. Clinical simulation: operations, engineering, and management. Burlington: Academic Press; 2008. p.541-50.

19. Kozlowski SW, DeShon RP. A psychological fidelity approach to simulation-based training: theory, research and principles. In: Sala E, Elliott LR, Schflett SG, Coovert MD, eds. Scaled worlds: development, validation, and applications. Burlington: Ashgate; 2004. p.75-99.

20. Brandão CFS, Carvalho-Filho MA, Cecilio-Fernandes D. Simulation centers and pedagogical planning: two sides of the same coin. Sci Med. 2018,28(1):28709. https://doi/ org/10.15448/1980-6108.2018.1.28709

21. The Lancet. COVID-19: protecting health-care workers. Lancet. 2020;395(10228):922. https://doi/org/10.1016/ S0140-6736(20)30644-9

22. Gardiner C, Veall J, Lockhart S. The use of UV fluorescent powder for COVID-19 airway management simulation training. Anaesthesia. 2020;75(7):964-5. https://doi/org/10.1111/anae.15089 